\title{
Estudio de la actividad antimicrobiana de películas delgadas de dióxido de titanio modificado con plata
}

\author{
William Vallejo ${ }^{1, *}$, Carlos Díaz-Uribe ${ }^{1}$, Karen Navarro ${ }^{1}$, Roger Valle $^{2,3}$, Jorge William Arboleda ${ }^{2}$ Eduard Romero $^{4}$ \\ ${ }^{1}$ Grupo de Fotoquímica y Fotobiología, Universidad del Atlántico, Barranquilla, Colombia \\ ${ }^{2}$ Facultad de Ciencias Básicas, Programa de Biología, Universidad del Atlántico, Barranquilla, Colombia \\ ${ }^{3}$ Programa de Doctorado en Medicina Tropical, Universidad de Cartagena, Cartagena, Colombia \\ ${ }^{4}$ Departamento de Química, Facultad de Ciencias, Universidad Nacional de Colombia, Bogotá, Colombia
}

\begin{abstract}
Resumen
Se estudió la actividad antimicrobiana de películas delgadas de dióxido de titanio $\left(\mathrm{TiO}_{2}\right)$ solo y modificado con plata. Las películas de $\mathrm{TiO}_{2}$ solo se sintetizaron con el método Doctor Blade y las modificadas con plata con el método de fotorreducción química bajo irradiación UV. Las propiedades de las películas se midieron por espectroscopia Raman y microscopía electrónica de barrido; también se determinó el efecto de la incorporación de las partículas de plata en las propiedades antimicrobianas de las películas de $\mathrm{TiO}_{2}$ frente a Staphylococcus aureus. Los resultados de la caracterización indicaron que las partículas de plata se aglomeraban sobre la superficie del $\mathrm{TiO}_{2}$ generando agregados del orden de los $200 \mathrm{~nm}$; en el análisis por espectroscopia Raman se vio que los agregados de plata depositados sobre las películas de $\mathrm{TiO}_{2}$ podrían presentar el fenómeno de resonancia de plasmones superficiales localizada. El ensayo preliminar de la actividad antimicrobiana indicó que la modificación de las películas de $\mathrm{TiO}_{2}$ con partículas de plata aumentó en 2,18 veces la eficiencia del proceso.
\end{abstract}

Palabras clave: dióxido de titanio, fotocatálisis, actividad antimicrobiana.

Antimicrobial activity of silver-modified titanium dioxide thin films

\begin{abstract}
We studied titanium dioxide $\left(\mathrm{TiO}_{2}\right)$ thin films and silver-modified titanium dioxide thin films antimicrobial activity. The $\mathrm{TiO}_{2}$ thin films were synthesized using Doctor Blade method. The synthesis of silver-modified films was done by chemical photoreduction under UV irradiation. Materials were characterized by Raman spectroscopy and scanning electron microscopy (SEM) measurements. We determined the antimicrobial effect against Staphylococcus aureus of the incorporation of silver particles on $\mathrm{TiO}_{2}$. Results indicated silver particles agglomerated on the surface of $\mathrm{TiO}_{2}$ generating aggregates of around $200 \mathrm{~nm}$. Raman spectroscopy suggested that silver aggregates may present the plasmon resonance superficial phenomenon. The antimicrobial activity assay indicated that the $\mathrm{TiO}_{2}$ films modification with silver increased by 2.18 times the efficiency of the process.
\end{abstract}

Key words: Titanium dioxide, photocatalysis, antimicrobial activity.

\section{Introducción}

La fotocatálisis heterogénea ha despertado considerable interés en la última década debido a sus aplicaciones en procesos de remediación ambiental (Park, et al., 2014). Muchos semiconductores sometidos a radiación UV y UVvis se han utilizado para la degradación de compuestos orgánicos e inorgánicos contaminantes y la inactivación de bacterias y virus en diferentes tipos de ambientes (Dizaj, et al., 2014). Uno de los semiconductores más estudiados es el dióxido de titanio $\left(\mathrm{TiO}_{2}\right)$, material inocuo que presenta una elevada resistencia a la fotocorrosión, es estable en soluciones acuosas, no es costoso, abunda en la naturaleza y posee una buena actividad fotocatalítica bajo irradiación ultravioleta (Malliga, et al., 2014).

El proceso fotocatalítico que usa $\mathrm{TiO}_{2}$ puede describirse de manera general como sigue:

$$
\begin{aligned}
& \mathrm{TiO}_{2}+\mathrm{h} "(\mathrm{E}>\mathrm{Eg}) \rightarrow \mathrm{TiO}_{2}\left(\mathrm{~h}_{\mathrm{vb}}{ }^{+} / \mathrm{e}_{\mathrm{cb}}{ }^{-}\right) \\
& \left(\mathrm{h}_{\mathrm{vb}}{ }^{+}\right)+\mathrm{H}_{2} \mathrm{O}_{\mathrm{ad}} \rightarrow \mathrm{OH}_{(\mathrm{ad})}^{\cdot}+\mathrm{H}^{+} \\
& \left(\mathrm{h}^{+}\right)+\mathrm{OH}^{-} \text {(ad) } \rightarrow \mathrm{OH}_{\text {(ad) }}^{\cdot} \\
& \left(\mathrm{h}^{+}\right)+\mathrm{P}_{(\mathrm{ad})} \rightarrow \mathrm{P}^{\cdot+}{ }_{(\mathrm{ad})} \\
& \mathrm{OH}_{(\text {ad) }}^{\cdot}+\mathrm{P}_{(\text {ad) }} \rightarrow(\mathrm{P}-\mathrm{OH})^{\cdot} \text { (ad) } \rightarrow \mathrm{P}^{++}{ }_{(\mathrm{ad})}+\mathrm{OH}^{-} \text {(ad) } \\
& \left(\mathrm{e}_{\mathrm{cb}}{ }^{-}\right)+\mathrm{O}_{2(\mathrm{ad})} \rightarrow \mathrm{O}_{2}{ }^{-} \text {(ad) }
\end{aligned}
$$

donde CB corresponde a la banda de conducción y VB a la banda de valencia del $\mathrm{TiO}_{2}$, y P es el contaminante (que en este trabajo fue Staphylococcus aureus). En los primeros

\footnotetext{
*Correspondencia:

William Vallejo, williamvallejo@mail.uniatlantico.edu.co

Recibido: 12 de octubre de 2015

Aceptado: 20 de enero de 2016
} 
instantes la radiación electromagnética con mayor energía que la brecha de bandas del $\mathrm{TiO}_{2}$ es absorbida sobre su superficie (ecuación 1) (Macwan, et al., 2011), lo cual genera un electrón en la banda de conducción y un hueco en la banda de valencia; luego, las moléculas de agua adsorbidas sobre la superficie del $\mathrm{TiO}_{2}$ generan radicales hidroxilo (ecuación 2). Los huecos también pueden generar radicales hidroxilo por la reacción directa con iones hidroxilos presentes en la solución (ecuación 3); al mismo tiempo, los electrones en la banda de valencia pueden generar radicales superóxido por la reacción con el oxígeno presente en la solución. Los radicales formados inician el proceso de degradación del contaminante (ecuaciones 5 y 6 ) (Peláez, et. al., 2012), en tanto que la presencia de oxígeno impide esta recombinación al atrapar electrones a través de la formación de aniones radicales superóxido (ecuación 6). La oxidación (ecuación 4) se ve favorecida por las moléculas que se absorben fácilmente en la fotocatálisis. Por otro lado, los radicales $\mathrm{OH}^{*}{ }_{\text {(ad) }}$ son capaces de reaccionar en la interfaz o difundirse lejos de la partícula de $\mathrm{TiO}_{2}$ (ecuación 5) con moléculas orgánicas en solución (Zhang, et al. 2013; Chorianopoulos, et al., 2011).

El $\mathrm{TiO}_{2}$ se ha empleado para la degradación de varias bacterias, en especial las Gram positivas como S. aureus (Pham \& Lee, 2015). Sin embargo, dos inconvenientes limitan la aplicación práctica de la tecnología basada en $\mathrm{TiO}_{2}$ : (a) este material solo es fotoactivo bajo irradiación ultravioleta $(\lambda<380 \mathrm{~nm})$, y (b) el proceso por lo general presenta poca eficiencia cuántica, con una rápida recombinación del par electrón-hueco fotogenerado. Para resolver estos inconvenientes se han explorado diferentes estrategias: (1) los métodos de síntesis del $\mathrm{TiO}_{2}$ se pueden variar y optimizar, y (2) se puede modificar la superficie del $\mathrm{TiO}_{2}$. Se ha reportado que la modificación del $\mathrm{TiO}_{2}$ con metales de transición (incluidos la plata, el platino, el rutenio y el paladio) disminuye el proceso de recombinación al formar heteroestructuras y nuevas interfaces que mejoran la eficiencia fotocatalítica (Gao \& Yang, 2015; Rodríguez, et al., 2010; Zhou, et al. 2011). Recientemente se ha encontrado que las partículas de plata tienen un amplio uso en el desarrollo de procesos biológicos y farmacéuticos, y se han usado como material de revestimiento para dispositivos médicos, materiales ortopédicos y ayudas tópicas para reparación de heridas, entre otros (Chen \& Schluesener, 2008; Russell \& Hugo, 1994; Mirzajani, et al., 2011). Igualmente, se han hecho estudios sobre la actividad antimicrobiana de las nanopartículas de plata frente a bacterias Gram positivas y Gram negativas en los que se han reportado buenos resultados (Shrivastava, et al., 2007).

En este trabajo se determinó el efecto de la incorporación superficial de partículas de plata sobre películas de $\mathrm{TiO}_{2}$ en sus propiedades antimicrobianas frente a $S$. aureus resistente a meticilina (SARM).

\section{Materiales y métodos}

\section{Síntesis de las películas delgadas de dióxido de titanio}

Para fabricar las películas de $\mathrm{TiO}_{2}$ se utilizó el método Doctor Blade. Se preparó una suspensión de dióxido de titanio mezclando 0,500 g de Degussa P-25 con 0,100 g de polietilenglicol macerados previamente en un mortero durante 15 minutos, a los que se añadieron $2 \mathrm{ml}$ de isopropanol con agitación constante durante una hora. La suspensión obtenida se depositó sobre un sustrato de vidrio (sílice) usando la técnica Doctor Blade (Ruani, et al., 2008; Wojcik, et al., 2010). Las películas se precalentaron a $90{ }^{\circ} \mathrm{C}$ durante 30 minutos y luego se calentaron a $500{ }^{\circ} \mathrm{C}$ durante una hora.

\section{Obtención de las películas de dióxido de titanio modificadas con plata}

Para depositar las partículas de plata sobre las películas de $\mathrm{TiO}_{2}$, se utilizó el método de fotorreducción química (Gao, et al., 2015). Para ello, las películas de $\mathrm{TiO}_{2}$ se sumergieron en una solución acuosa de nitrato de plata $(0,025 \mathrm{~g}$ de $\mathrm{AgNO}_{3}$ en $5 \mathrm{ml}$ de agua desionizada), y luego se irradió con una lámpara UV con un máximo de emisión de 260 nm y una intensidad de $30 \mathrm{~W}$ durante 30 minutos. Después del proceso de fotorreducción el color superficial de las películas de $\mathrm{TiO}_{2}$ cambió de blanco a café grisáceo.

\section{Caracterización de los materiales}

Las propiedades de composición y morfología de los materiales se estudiaron mediante espectroscopia Raman en un equipo DXR $780 \mathrm{~nm}$ LASER y microscopía electrónica de barrido en un equipo SEM Tescan VEGA3 SB equipado para la espectroscopia de energía dispersiva de rayos X (EDS).

\section{Ensayo preliminar de la actividad antimicrobiana}

Para el ensayo preliminar de la actividad antimicrobiana de los materiales se empleó la metodología de unidades formadoras de colonia (UFC) (Shrivastava, et al., 2007). Para ello se inocularon las cepas de SARM en 30 $\mathrm{ml}$ de caldo nutritivo estéril, y se dejaron en incubación y agitación durante más de 12 horas. Los cultivos de SARM se prepararon en tubos Falcon con una absorbancia conocida $(0,05)$. Se introdujo en cada tubo un tratamiento, es decir, una película del respectivo material, lo cual se hizo por triplicado para cada tratamiento. Además, se usó gentamicina como control positivo y se dejó crecer un cultivo sin ningún tratamiento como control negativo. Se hizo una dilución de $10^{-7}$ y se usó la técnica de cuenta en placa. Luego se sometieron los tubos Falcon a irradiación UV durante 30 min con una lámpara de $360 \mathrm{~nm}$ de longitud de onda máxima. Los tubos se sometieron a incubación y agitación durante 12 horas y nuevamente se hizo la dilución y el conteo en placa. Por último, se hizo un conteo electrónico de las UFC encontradas en las placas. En la Figura 1 se muestra el esquema general de la metodología aquí descrita. 


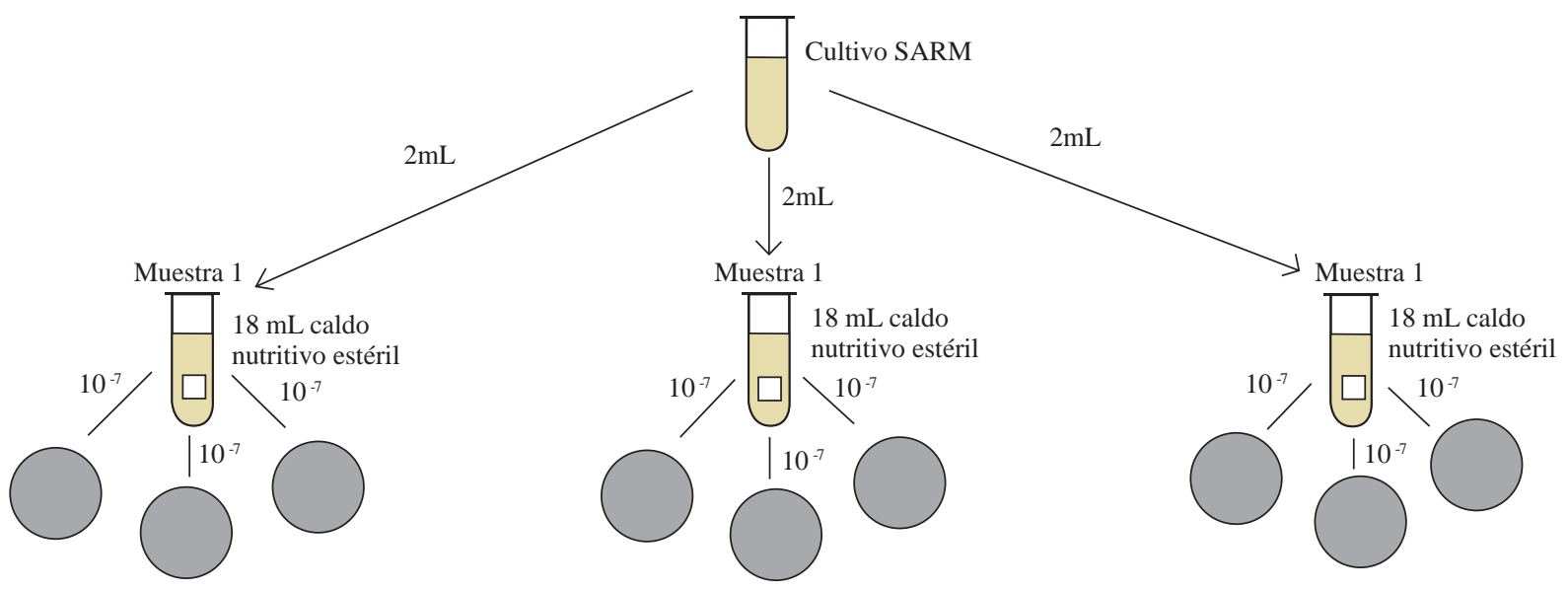

Irradiación UV por 30 min de los tubos con los tratamientos. Luego los tubos son incubados y agitados por 12 horas. Se repite el procedimiento anterior.
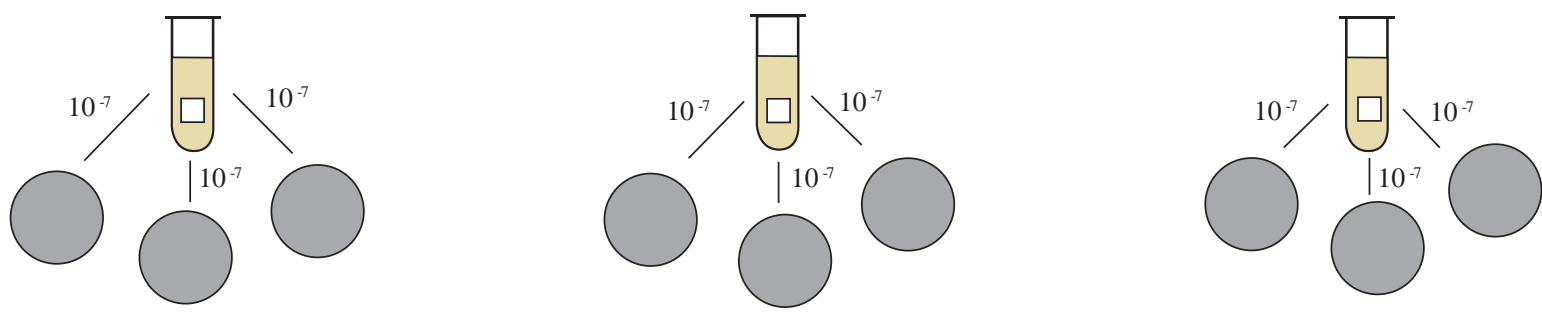

Figura 1. Esquema general de la metodología de unidades formadoras de colonia utilizada para los ensayos de inhibición del crecimiento de Staphylococcus aureus bajo irradiación UV

\section{Resultados y discusión}

\section{Caracterización de los materiales mediante espectrometría Raman}

En la Figura 2 se muestra el espectro Raman obtenido para las películas de $\mathrm{TiO}_{2}$ y de este modificado con plata $\left(\mathrm{TiO}_{2}-\right.$ Ag). Las películas de $\mathrm{TiO}_{2}$ presentaron los picos Raman característicos de la fase anatasa y los modos de simetría de vibración para la fase anatasa indicados: $\mathrm{E}_{1 \mathrm{~g}}$ para las señales localizadas a $144 \mathrm{~cm}^{-1}, 398 \mathrm{~cm}^{-1}$ y $520 \mathrm{~cm}^{-1}$, y E para la señal localizada a $639 \mathrm{~cm}^{-1}$, asignaciones que se corresponden con las reportadas en la literatura (Vishwas, et al., 2012; Liu, et al., 2001). En el espectro Raman de las peliculas modificadas de $\mathrm{TiO}_{2}-\mathrm{Ag}$ se pudo observar que la intensidad de las señales aumentó en comparación con las películas de $\mathrm{TiO}_{2}$ Degussa P25; este fenómeno se conoce como incremento superficial de espectroscopia Raman, y se caracteriza por el aumento en varios órdenes de magnitud de la intensidad de las señales en el espectro Raman por efecto de especies adsorbidas sobre la superficie del metal (Ma, et al., 2011); el fenómeno fisicoquímico indica que algunas nanopartículas de metales nobles (oro, plata, paladio y platino) pueden generar una intensa resonancia plasmónica superficial cuando son adsorbidas sobre semiconductores (Fang, et al., 2015). El incremento en la intensidad de las señales espectroscópicas Raman para las películas de $\mathrm{TiO}_{2}$-Ag sugiere que es posible que las partículas de plata 'fotoadsorbidas' sobre la superficie

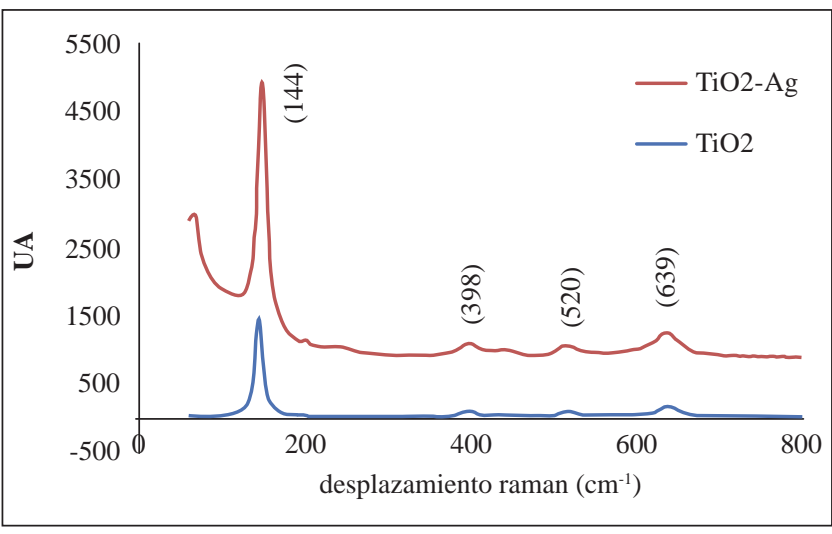

Figura 2. Espectro Raman de películas sintetizadas de dióxido de titanio solo y modificado con plata

del $\mathrm{TiO}_{2}$ se presenten agrupadas a escala nanométrica, esta observación que se verifica mediante el análisis por microscopia electrónica de barrido.

\section{Estudio de la morfología de los materiales}

El estudio de la morfología y la composición química de los materiales obtenidos en esta investigación se hizo mediante microscopia electrónica de barrido y EDS. La Figura 3a muestra la imagen de microscopia electrónica de barrido de las películas de $\mathrm{TiO}_{2}$ y $\mathrm{TiO}_{2}$-Ag. La Figura 3 a muestra que las películas de $\mathrm{TiO}_{2}$ estaban conformadas por microagregados 

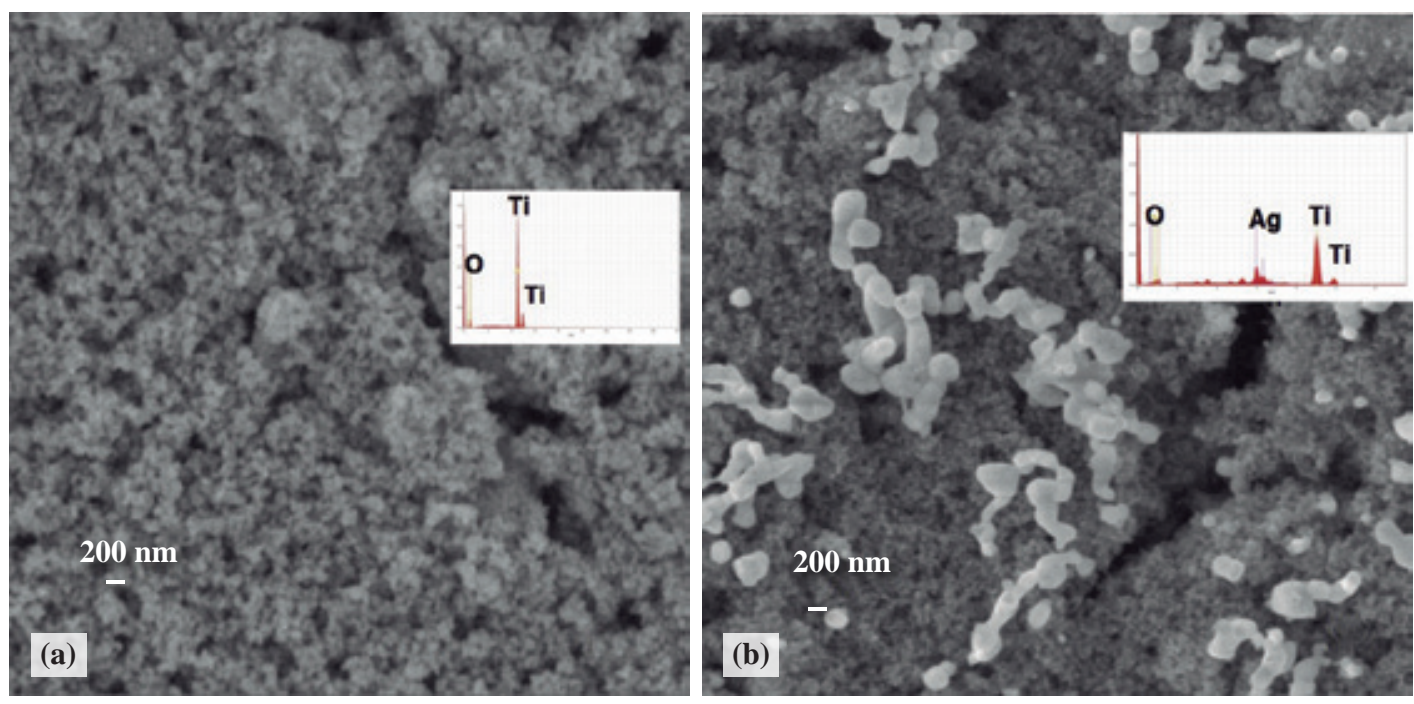

Figura 3. Imágenes de microscopia electrónica de barrido de: (A) una película de dióxido de titanio, y (B) una película de dióxido de titanio modificado con plata. En cada imagen se muestra el análisis mediante espectroscopia de energía dispersiva de rayos X.

con un estrecho margen de tamaño del orden de los 50 nm; se observó la morfología típica del $\mathrm{TiO}_{2}$ Degussa P25. Mediante el análisis por EDS se verificó la composición del material utilizado para la obtención de las películas. Las imágenes obtenidas evidencian baja uniformidad superficial en las películas (Clavijo, 2013).

En la Figura $3 b$ se muestra el análisis morfológico de la película de $\mathrm{TiO}_{2}$ con nanopartículas de plata. Se pudo observar un cambio significativo en la morfología de la superficie del $\mathrm{TiO}_{2}$; la imagen SEM permitió diferenciar las partículas de plata depositadas (Cueto \& Sánchez, 2010). El análisis SEM indicó que las partículas fotorreducidas se aglomeraron alcanzado tamaños del orden de los $200 \mathrm{~nm}$. Mediante el análisis por EDS se verificó que los aglomerados observados en la superficie del $\mathrm{TiO}_{2}$ correspondían a partículas de plata (Figura 3b).

\section{Ensayo preliminar de la actividad antimicrobiana}

Con el ánimo de verificar la posible aplicación de los materiales obtenidos, se hizo un ensayo de la actividad antimicrobiana contra SARM bajo irradiación UV. En la Figura 4 se muestra el porcentaje de inhibición de las películas de $\mathrm{TiO}_{2}$ y de $\mathrm{TiO}_{2}$-Ag. Se pudo observar que las películas de $\mathrm{TiO}_{2}$-Ag presentaron un mayor porcentaje de inhibición (35\%) que las películas de $\mathrm{TiO}_{2}$ sin modificar $(16 \%)$. Este resultado puede atribuirse a dos fenómenos: (a) los agregados de plata depositados sobre la superficie pueden acumular carga y aceptar electrones fotogenerados al interior del semiconductor, la acumulación de electrones puede disminuir la recombinación de portadores y favorecer su transferencia a otras especies para la generación de especies reactivas de oxígeno ó; (b) un aumento en la generación de portadores de carga, ya sea por influencia del campo magnético inducido por el metal incorporado o por

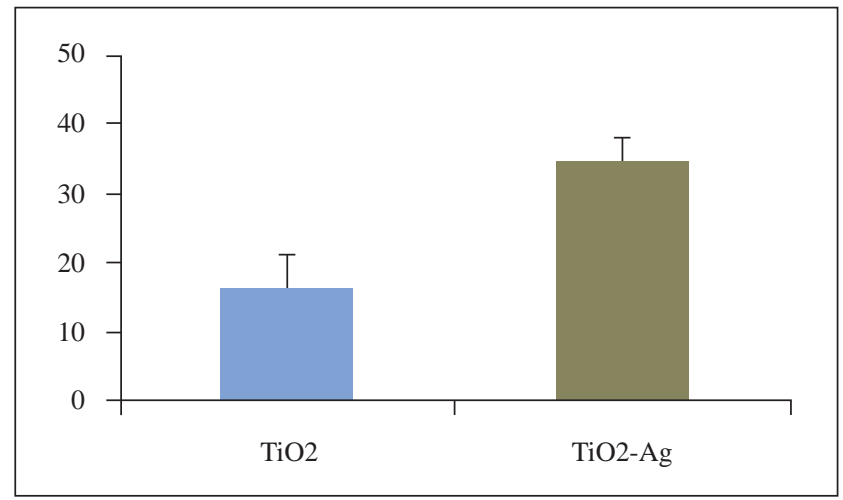

Figura 4. Porcentajes de inhibición del crecimiento de Staphylococcus aureus sobre películas de dióxido de titanio solo y modificado con plata bajo irradiación UV.

el calentamiento térmico ocasionado por la transferencia de carga desde el material adsorbido (Zielińska-Jurek, et al., 2015; Stroyuk, et al., 2010). La segunda de estas hipótesis cobra fuerza debido a los resultados obtenidos en la caracterización por Raman y por SEM; el efecto de incremento superficial de la espectroscopia Raman y la presencia de agregados del orden de los $200 \mathrm{~nm}$ sugieren que esta podría ser una de las razones del incremento de la actividad antimicrobiana.

Según André, et al. (2015), las partículas de plata exhiben oscilación colectiva de sus electrones en la banda de conducción, lo que se conoce como resonancia de plasmones superficiales localizada, y se sugiere que la asociación entre el semiconductor y el metal cambia la eficacia y el mecanismo de la actividad antimicrobiana de cada uno, lo que coincide con los resultados obtenidos en esta investigación. 


\section{Conclusiones}

En este trabajo se fabricaron películas delgadas de $\mathrm{TiO}_{2}$ y de $\mathrm{TiO}_{2}-\mathrm{Ag}$ usando la técnica Doctor Blade; las partículas de plata se depositaron mediante fotorreducción química. El análisis morfológico indicó que se formaron aglomerados de plata de un tamaño promedio de $200 \mathrm{~nm}$ en la superficie del semiconductor. Los resultados de la EDS demostraron la presencia de partículas de plata en la superficie del $\mathrm{TiO}_{2}$. El análisis espectroscópico sugiere que las películas de $\mathrm{TiO}_{2}-\mathrm{Ag}$ pueden presentar efectos de resonancia plasmónica superfical. Por último, los ensayos de inactividad antimicrobiana contra SARM indicaron que la modificación de las películas de $\mathrm{TiO}_{2}$ con partículas de plata aumentó en 2,18 veces la eficiencia del proceso.

\section{Agradecimientos}

Los autores agradecen la colaboración del Doctor Mikel Hurtado por su apoyo en la interpretación de los espectros Raman, así como el apoyo de la Universidad del Atlántico en la ejecución de este trabajo. Los resultados corresponden al proyecto presentado en la Convocatoria IMPACTO CARIBE 2014, convocado por la Universidad del Atlántico (Resolución 002627 del 3 de marzo de 2015 - Proyecto número 24).

\section{Conflicto de intereses}

Los autores declaran que no tienen conflicto de intereses.

\section{Bibliografía}

André, R. S. Zamperini, C. A. Mima, E. G. Longo, V. M. Albuquerque, A. R. Sambrano, J. R. Machado, A. L. Vergani, C. E. Hernandes, A. C. Varela, J. A. \& Longo, E. (2015). Antimicrobial activity of $\mathrm{TiO}_{2}$ : Ag nanocrystalline heterostructures: Experimental and theoretical insights, Chemical Physics. 459: 87-95.

Chorianopoulos, N. G. Tsoukleris, D. S. Panagou, E. Z. Falaras, P. \& Nychas, G. J. (2011) Use of titanium dioxide $\left(\mathrm{TiO}_{2}\right)$ photocatalysts as alternative means for Listeria monocytogenes biofilm disinfection in food processing. Food Microbiology. 28: 164-170.

Chen \& Schluesener. (2008) Nanosilver: A nanoproduct in medical application. Toxicology Letters. 176: 1-12.

Clavijo, J. (2013) Caracterización de materiales a través de medidas de microscopía electrónica de barrido. Revista Elementos. 3: 134-146.

Cueto, L. F. Sánchez, E. M. (2010). Caracterización de películas delgadas de $n-\mathrm{TiO}_{2}$ modificadas con nanoparticulas de plata. $19^{\circ}$ Congresso Brasileiro de Engenharia e Ciência dos Materiais - CBECiMat, Campos do Jordão, SP, Brasil.

Dizaj, S. M. Lotfipour, F. Barzegar-Jalali, M. Zarrintan, M. H. \& Adibkia, K. (2014) Antimicrobial activity of the metals and metal oxides nanoparticles. Materials Science \& Engineering C. 44: 278-284.

Fang, H. Xing, C. Liu, L. Zhao, Y. M. \& Jun, H. (2015). Recyclable three-dimensional Ag nanoparticle-decorated
$\mathrm{TiO}_{2}$ nanorod arrays for surface-enhanced Raman scattering. Biosensors and Bioelectronics. 64: 434-441.

Gao, F. Yang, Y. \& Wang, T. (2015). Preparation of porous $\mathrm{TiO}_{2} /$ $\mathrm{Ag}$ heterostructure films with enhanced photocatalytic activity. Chemical Engineering Journal. 270: 418-427.

Liu, Y. Liu, C. Zhang, Z. Y. \& Wang, C. Y. (2001). The surface-enhanced Raman scattering effects of composite nanocrystals of $\mathrm{Ag}-\mathrm{TiO}_{2}$. Spectrochimica Acta Part A: Molecular and Biomolecular Spectroscopy. 57: 35-39.

Malliga, P. Pandiarajan, J. Prithivikumaran, N. \& Neyvasagam, K. (2014). Influence of film thickness on structural and optical properties of sol-gel spin coated $\mathrm{TiO}_{2}$ thin film. Journal Applied Physics. 6: 22-28.

Macwan, D. P. Dave, P. N. \& Chaturvedi, S. (2011). A review on nano- $\mathrm{TiO}_{2}$ sol-gel type syntheses and its applications. Journal of Materials Science. 46: 3669-3686.

Mirzajani, F. Ghassempour, A. Aliahmadi, A. \& Esmaeili, M. A. (2011). Antibacterial effect of silver nanoparticles on Staphylococcus aureus. Research in Microbiology. 162: 542-549.

Ma, S. Livingstone, R. Zhao, B. \& Lombardi, J. (2011). Enhanced Raman spectroscopy of nanostructured semiconductor phonon modes. Journal Physical Chemistry Letters. 2: 671-674.

Park, E. J. Jeong, B. Jeong, M. G. \& Kim Y. D. (2014). Synergetic effects of hydrophilic surface modification and N-doping for visible light response on photocatalytic activity of $\mathrm{TiO}_{2}$. Current Applied Physics. 14: 300-305.

Pelaez, M. Nolan, N. T. Pillai, S. C. Seery, M. K. Falaras, P. Kontos, A. G. Dunlop, P. Hamilton, J. Byrne, J. O'Schea, K. Entezari, M. H. \& Dionysious D. (2012). A review on the visible light active titanium dioxide photocatalysts for environmental applications. Applied Catalysis B: Environmental. 125: 331-349.

Pham, T. D. \& Lee, B. K. (2015). Disinfection of Staphylococcus aureus in indoor aerosols using $\mathrm{Cu}-\mathrm{TiO}_{2}$ deposited on glass fiber under visible light irradiation. Journal of Photochemistry and Photobiology A: Chemistry. 307: 16-22.

Rodríguez, V. Algaro, S. O. Torres, L. M. Chod, S. H. \& Lee, S. W. (2010). Silver- $\mathrm{TiO}_{2}$ nanocomposites: Synthesis and harmful algae bloom UV-photoelimination. Applied Catalysis B: Environmental. 98: 229-234.

Russell, A. D. Path, F. R. Si, F. P. \& Hugo, W. B. (1994) Antimicrobial activity and action of silver. Progress in Medicinal Chemistry. 31: 351-370.

Ruani, G. Ancora, C. Corticelli, F. Dionigi, C. \& Rossi C. (2008). Single-step preparation of inverse opal titania films by the Doctor Blade technique. Solar Energy Materials \& Solar Cells. 92: 537-542.

Shrivastava, S. Bera, T. Roy, A. Singh, G. Ramachandrarao, P. \& Dash, D. (2007). Characterization of enhanced antibacterial effects of novel silver nanoparticles. Nanotechnology. 18: 225103. 
Stroyuk, O. L. Kuchmiy, S. Y. Kryukov, A. I. \& Pokhodenko V. D. (2010). Semiconductor catalysis and photocatalysis on the nanoscale. Nova Science Publishers, Inc., New York. p.183.

Vishwas, M. Rao, K. N. \& Chakradhar, R. P. S. (2012). Influence of annealing temperature on Raman and photoluminescence spectra of electron beam evaporated $\mathrm{TiO}_{2}$ thin films. Spectrochimica Acta Part A: Molecular and Biomolecular Spectroscopy. 99: 33-36.

Wojcik, I. Senadeera, R. Wojcik, P. J. Silva, L. B. Doria, G. Baptista, P. Aguas, H. Fortunato, E. \& Martins, R. (2010). Inkjet printed and "doctor blade" $\mathrm{TiO}_{2}$ photodetectors for DNA biosensors. Biosensors and Bioelectronics. 25: 1229-1234.
Zielińska-Jurek, A. Wei, Z. Wysocka, I. Szweda, P. \& Kowalska, S. (2015). The effect of nanoparticles size on photocatalytic and antimicrobial properties of $\mathrm{Ag}-\mathrm{Pt} / \mathrm{TiO}_{2}$ photocatalysts. Applied Surface Science. 353: 317-325.

Zhou, W. Du, G. Hu, P. Yin, Y. Li, J. Yu, J. Wang, G. Wang, J. Liu, H. Wang, J. \& Zhang, H. (2011). Nanopaper based on $\mathrm{Ag} / \mathrm{TiO}_{2}$ nanobelts heterostructure for continuous-flow photocatalytic treatment of liquid and gas phase pollutants. Journal of Hazardous Materials. 197: 19-25.

Zhang, Y. Lee, M. An, S. Sinha-Ray, S. Khansari, S. Joshi, B. Hong, S. Hong, J. Kim, J. Pourdeyhimi, B. Yoon, S. \& Yarin, A. (2013). Antibacterial activity of photocatalytic electrospun titania nanofiber mats and solution-blown soy protein nanofiber mats decorated with silver nanoparticles. Catalysis Communications. 34: 35-40. 